\title{
Pain rather than self-reported sedentary time explains variation in perceived health and activity limitation in persons with rheumatoid arthritis: a cross sectional study in Sweden
}

\author{
Ingrid Demmelmaier ${ }^{1}$ (1) $~$ Pernilla Åsenlöf ${ }^{2}$ Patrick Bergman ${ }^{3} \cdot$ Birgitta Nordgren $^{1}$ • \\ Christina H. Opava ${ }^{1,4}$
}

Received: 31 August 2016 / Accepted: 21 December 2016 / Published online: 25 January 2017

(c) The Author(s) 2017. This article is published with open access at Springerlink.com

\begin{abstract}
To investigate (1) the amount of self-reported time spent sedentary among a large cohort of persons with rheumatoid arthritis (RA), and (2) the contribution of sedentary time to explain perceived health and activity limitation in RA beyond that of previously known correlates. This cross-sectional study used data from a postal questionnaire and the Swedish Rheumatology Quality registers (SRQ). The International Physical Activity Questionnaire was used to assess sedentary time (sitting) and moderate, vigorous and walking activity (MVPA). Sociodemographics, pain, fatigue, fear-avoidance beliefs, anxiety/ depression, disease duration, MVPA and sedentary time were included in multiple regression models with perceived health (Visual Analogue Scale 0-100) and activity limitation (Stanford Health Assessment Questionnaire) as dependent variables. Results: In all 3152 (59\%) of 5391 persons identified as eligible from the SRQ, responded to the questionnaire. 2819 individuals with complete data on all study variables were analysed. Mean time (SD) spent sedentary was 257 (213) minutes per day. Sedentary time did not contribute significantly to explain perceived health
\end{abstract}

I. Demmelmaier and P. Åsenlöf equally contributed to this study.

Ingrid Demmelmaier

ingrid.demmelmaier@ki.se

1 Division of Physiotherapy, Department of Neurobiology, Care Sciences and Society, Karolinska Institutet, 23100, Alfred Nobels Allé 23, 14183 Huddinge, Sweden

2 Department of Neuroscience, Uppsala University, Uppsala, Sweden

3 School of Education, Psychology and Sports Science, Linnaeus University, Kalmar, Sweden

4 Department of Rheumatology, Karolinska University Hospital, Stockholm, Sweden and only minimally to explain activity limitation. Instead, variation was mainly explained by pain; for perceived health $($ Beta $=0.780, p<0.001)$ and for activity limitation $($ Beta $=0.445, p<0.001)$.The results indicate a non-significant role of sedentary time and a need for increased focus on pain in the management of RA. Future studies should use prospective designs and objective assessment methods to further investigate the associations between sedentary time and health outcomes in persons with RA.

Keywords Rheumatoid arthritis · Sedentary time · Sitting $\cdot$ Disability $\cdot$ Health $\cdot$ Multiple regression

\section{Introduction}

'Sedentary time' refers to the time spent in activities that do not increase energy expenditure substantially above the resting level (1.0-1.5 Metabolic Equivalent of Task, METs), such as watching computer screens or television, and is often measured by time spent in a sitting or reclining position [1,2]. Evidence is accumulating on the negative effects of prolonged sedentary time, pointing to increased risk of cardiovascular disease, metabolic syndrome, and mortality in various populations [3-5]. Studies on physiological mechanisms indicate that inactivity and muscle disuse may cause low-grade chronic systemic inflammation [6], putting sedentary persons with inflammatory diseases such as rheumatoid arthritis (RA) at particular risk.

Impact and levels of sedentary time in populations with RA have been minimally explored. However, one study on patients with RA reported lower bone mass among those who spent prolonged periods of time sedentary compared to those who did not [7], and two recent accelerometer studies found persons with RA to spend 
on average almost $10 \mathrm{~h}$ per day sedentary $[8,9]$. These figures are to be compared with studies conducted on the general population [10-13], reporting a mean daily sedentary time of $8-9 \mathrm{~h}$, and on populations with long-term conditions such as stroke (13 h) [14] and osteoarthritis (10 h) [15].

Functioning is recognized as an important outcome in RA [16]. Based on the International Classification of Disability, Functioning and Health (ICF), the ICF core set for RA includes patient-reported outcomes that are used to complement medical measures [17]. Two crucial patient-reported outcomes, reflecting the over-arching ICF concepts of health and disability in RA, are perceived health and activity limitation [17].

Despite the accumulating evidence for the negative effects of prolonged sedentary time, its individual contribution to explaining health outcomes in RA is unknown. Previously identified predictors of health outcomes include pain, fatigue, depression, disease activity, radiographic progression [18, 19], physical activity [20], gender, and age [21]. The present study aimed to investigate (1) the amount of self-reported time spent sedentary among a large cohort of persons with RA, and (2) the contribution of self-reported sedentary time to explain perceived health and activity limitation in RA beyond that of a number of previously identified correlates.

\section{Methods}

\section{Design and selection}

We applied a cross-sectional design using the Swedish Rheumatology Quality Registers (SRQ) for selection. Criteria for inclusion were diagnosis of RA, an age of under 75 years, and a Stanford Health Assessment Questionnaire (HAQ) disability index score of $\leq 2$. We approached 5391 individuals meeting the inclusion criteria of whom $3152(58.5 \%)$ consented to participate. The selection procedure was the first step in the recruitment process for a physical activity intervention [22], thus motivating the inclusion criteria on age and disability index score. Participants were sent a letter containing information about the study along with the study questionnaire and provided their consent by returning the completed questionnaire. Non-responders were younger, had shorter disease duration, and were slightly more affected by their RA at their most recent medical examination as compared to responders [23]. Our study group comprised 2819 individuals with complete data on all study variables.

\section{Measures}

\section{Dependent variables}

Perceived health was rated on a $100 \mathrm{~mm}$ visual analogue scale (VAS) from 0 (excellent health) to 100 (worst imaginable health), with high values indicating increased disease impact on health. The scale is considered valid and reliable in RA populations [24].

Activity limitation was assessed by the Stanford Health Assessment Questionnaire (HAQ) [25] with 20 items about daily activities performed during the past week, such as dressing and grooming, getting up, eating, taking care of personal hygiene, reaching, gripping, walking, and other common activities. Ratings were made on a four-point scale from 'With no difficulty' $(=0)$ to 'Unable to perform' $(=3)$. A total score between 0 and 3.0 was then calculated, where high values indicated increased activity limitation. The Swedish HAQ is considered valid and reliable in RA populations [26].

\section{Independent variables}

Background data on age, gender, education, income, number of adults in the household, pain (VAS 0-100), fatigue (VAS 0-100), fear-avoidance beliefs with a modified FearAvoidance Beliefs Questionnaire (0-24) [27], and anxiety/ depression with one EuroQoL-5 Dimension (EQ-5D) [28] item (0-3) were collected via questionnaires. Data on disease duration were retrieved from the SRQ.

Moderate-to-vigorous physical activity (MVPA) was assessed by six items from the International Physical Activity Questionnaire (IPAQ) short form, which asked questions about the frequency and duration of vigorousintensity, moderate-intensity, and walking physical activity, respectively. The responses were coded using established methods for the IPAQ [29]. Time spent in vigorous, moderate, and walking activity was weighted by the energy expended for these categories of activity to produce MET minutes of physical activity. The IPAQ has demonstrated acceptable test-retest reliability and criterion-related validity compared with accelerometers in the general population [30], and a mix of overestimation and underestimation of energy expenditure compared with accelerometers in an RA sample [31].

Sedentary time was assessed by the following item from the IPAQ short form: "During the past 7 days, how much time did you usually spend sitting on a weekday?" Responses were given in hours and minutes. The test-retest reliability of this item has been reported as good (Spearman rho values $>0.7$ in four country samples) and validity against accelerometers as acceptable [32]. 


\section{Data management and statistics}

Respondents' level of education was originally gathered in five categories: basic education, college, less than 2 years of university, three or more years of university, and other education. The two university education categories were subsequently collapsed into one, and the remaining alternatives into another. The number of adults in the household was initially recorded in four categories: living alone, living with one adult, living with two adults, and living with three or more adults. This was later modified to a simple yes/no answer as to whether there were other adults in the household. The variable of anxiety/depression was, due to very few study participants reporting extreme problems, dichotomized from three levels (no problems, some problems, and extreme problems) by collapsing the two last categories into one. The MVPA variable is the sum of the weighted MET minutes from the variables walking (the time reported times 3.3 MET), moderate physical activity (4 MET) and vigorous physical activity (8 MET).

The study sample is described using mean and standard deviation (SD) for continuous variables and by proportions for categorical variables. Independent sample $t$ test and chi-square tests were used to compare the study sample with the participants that were excluded due to incomplete data.

The independent contributions of MVPA and sedentary time to the explanation of variation in activity limitation and perceived health, respectively, were analysed in a series of multiple linear regression models. The models were built by first entering the background variables (age, gender, education, income, number of adults in the household, anxiety/depression, pain, fatigue, fearavoidance beliefs, and disease duration). Second, MVPA was entered and, in the final step, sedentary time. The variables were entered in this order to assess any additional contribution of sedentary time in addition to previously identified correlates. No signs of multicollinearity were observed; however, due to unsatisfactory residuals, the dependent variable 'perceived health' was transformed using the square root. The MVPA variable is the sum of the weighted MET minutes from the variables walking (the time reported $\times 3.3$ MET), moderate physical activity (the time reported $\times 4$ MET) and vigorous physical activity (the time reported $\times 8$ MET).

All data were analysed using IBM Statistical Package for the Social Sciences (SPSS) for Windows version 21. Alpha levels were set to 0.05 for the multiple regression models and the individual contribution of each independent variable.

\section{Results}

\section{Participants}

The sample included 2819 individuals with complete data on all study variables. Descriptives and a comparison with participants excluded due to incomplete data are presented in Table 1.

Compared to individuals with incomplete data $(n=333)$, our sample was younger, had higher education and income, reported less pain and less fatigue, performed more MVPA, spent more time sedentary, had better perceived health and experienced less activity limitation.

\section{Sedentary time}

Self-reported sedentary time was mean $257(\mathrm{SD}=213)$ minutes per day.

\section{Contribution of sedentary time to explaining variation in perceived health and activity limitation}

The outcomes of the regression models for explanations of perceived health and activity limitation are presented in Tables 2 and 3, respectively. The total amount of explained variance for each model is given, as well as the contribution of each independent variable.

\section{Discussion}

This study provides new knowledge about the amount of time spent sedentary in a large sample of persons with RA, and demonstrated that self-reported sedentary time did not contribute substantially to explain the variation in perceived health and activity limitation. Rather, the major parts of variation were explained by self-reported pain, indicating that pain overrides the influence that sedentary time might have on both outcomes.

Spending approximately $4 \mathrm{~h}$ per day sedentary during waking hours is surprisingly little compared to results from other studies using the single IPAQ item on sitting. One study on general population samples from 20 countries reported sedentary time as median $5 \mathrm{~h}$ per day [10]. Another study on persons with multiple sclerosis ( $84 \%$ female, ambulatory and one-month relapse-free) reported a mean of $7.5 \mathrm{~h} /$ day [33], and a recent review found average levels falling between 8.5 and $9.6 \mathrm{~h} /$ day in older adults [34]. Our data did not provide any explanation for the low amount of sedentary time in the adult RA population, but this may be found in the operationalization of sedentary time as sitting time [35]. People living with RA may go earlier to bed at night due to fatigue, and 
Table 1 Descriptives of the study sample $(n=2819)$ compared to participants excluded due to incomplete data $(n=333)$

\begin{tabular}{|c|c|c|c|}
\hline & Study sample $(n=2819)$ & Excluded $(n=333)$ & $p$ \\
\hline Age, mean \pm SD & $59 \pm 11$ & $64 \pm 10$ & $<0.001$ \\
\hline Gender, $n(\%)$ & & & 0.480 \\
\hline Female & $2059(73)$ & $250(75)$ & \\
\hline Male & $760(27)$ & $84(25)$ & \\
\hline Education, $n(\%)$ & & & $<0.001$ \\
\hline University & $953(34)$ & $72(22)$ & \\
\hline Not university & $1866(66)$ & $262(78)$ & \\
\hline Income (below or above Swedish average), $n(\%)$ & & & $<0.001$ \\
\hline Below & $1453(51)$ & $178(73)$ & \\
\hline Above & $1366(49)$ & $65(27)$ & \\
\hline Other adults in household, $n(\%)$ & & & 0.613 \\
\hline Yes & $2137(76)$ & $249(75)$ & \\
\hline No & $682(24)$ & $82(25)$ & \\
\hline Anxiety/depression (item 5 EQ-5D), $n(\%)$ & & & 0.400 \\
\hline Yes & $1006(36)$ & $127(62)$ & \\
\hline No & $1813(64)$ & $207(38)$ & \\
\hline Pain (VAS 0-100) ${ }^{\mathrm{a}}$, mean \pm SD & $30 \pm 24$ & $35 \pm 27$ & 0.001 \\
\hline Fatigue $(\text { VAS } 0-100)^{\mathrm{a}}$, mean \pm SD & $39 \pm 26$ & $42 \pm 28$ & 0.001 \\
\hline Fear-avoidance beliefs (mFABQ $0-24)^{\mathrm{a}}$, mean \pm SD & $8 \pm 6$ & $8 \pm 5$ & 0.481 \\
\hline Disease duration (years), mean \pm SD & $4 \pm 5$ & $4 \pm 3$ & 0.532 \\
\hline MVPA (MET min/day), mean \pm SD & $2544 \pm 2918$ & $1735 \pm 2615$ & $<0.001$ \\
\hline Sedentary time (min/day), mean \pm SD & $257 \pm 213$ & $160 \pm 185$ & $<0.001$ \\
\hline Perceived health (VAS 0-100) ${ }^{\mathrm{a}}$ & $30+24$ & $34+27$ & 0.007 \\
\hline Activity limitation (HAQ 0-3) & $0.6+0.6$ & $0.7+0.6$ & 0.001 \\
\hline
\end{tabular}

EQ-5D EuroQoL-5 Dimension, VAS Visual Analogue Scale, $M V P A$ Moderate-to-Vigorous Physical Activity, $m F A B Q$ modified Fear-Avoidance Beliefs Questionnaire, MET Metabolic Equivalent of Task, HAQ Stanford Health Assessment Questionnaire

$p$ values are based on independent sample $t$ test and Chi-square-test

${ }^{a}$ High values indicates worse health and more fear-avoidance beliefs

remain longer in bed or even go back to bed due to morning stiffness and pain [36], leaving less time for daytime activities including sitting. Another explanation may be that spending much time sitting is not necessary during periods of remission [36], which may have been the case for some participants.

The explanatory value of time spent sedentary in our study was unexpectedly low, considering the amount of research reporting the negative impact of a sedentary lifestyle on health, as well as its contribution to the burden of chronic disease worldwide [37, 38]. Sedentary time has also been found to be negatively associated with functioning in osteoarthritis [15, 39]. The contribution of MVPA to explain variations in both perceived health and activity limitation in the present study was small. We had expected MVPA to make a greater contribution, as recent reviews have reported positive health outcomes of MVPA in RA [40], other rheumatic diseases [41] and osteoarthritis [39, 42]. However, it should be acknowledged that differences in design and methods, e.g. self-reports versus objective assessment of physical activity may explain part of the disparity in findings.

Pain was by far the strongest explanatory variable of variation for both outcomes in the present study, particularly regarding perceived health. The results thus indicate that pain overrides the influence of all other included independent variables, highlighting an urgent need to address pain in RA treatment and rehabilitation. Since pain is one cardinal symptom in RA, described as a combined effect of nociceptive signals during active joint inflammation flares and generalized, central pain mechanisms [43, 44], it is important to consider both mechanisms in RA management. Multidisciplinary programmes for pain rehabilitation have been developed to address the complex nature of longterm pain [45], but have not been fully implemented within rheumatology. Further research is needed to implement and evaluate multidisciplinary pain programmes, combining pharmacological and non-pharmacological interventions, adapted to the individual needs of each patient in RA populations [46]. 


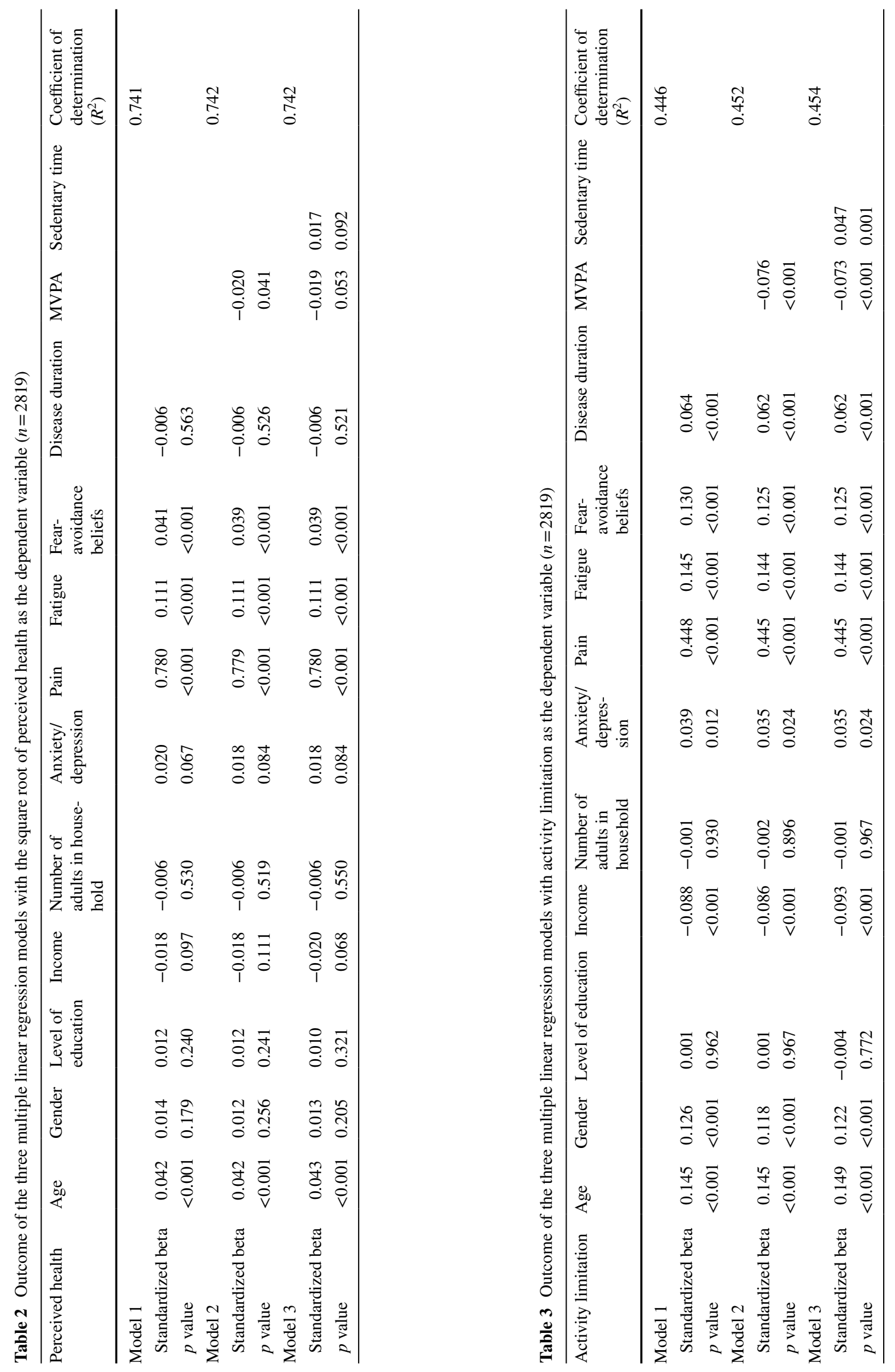




\section{Methodological strengths and limitations}

One strength of the study is the operational definition of sedentary time as a construct separate from physical activity, with these being scored as separate entities. Separating the two constructs into different behaviours is significant, as they may be determined by different bio-psycho-social factors. Different interventions will be needed depending on whether the aim is to decrease sedentary behaviours or to increase physical activity behaviours. Other strengths include the large and well-defined sample, made possible by the use of the SRQ for selection of participants.

Limitations refer to the use of self-reported data and its inherent risk of recall bias. Another limitation is the operational definition of sedentary behaviour as self-reported time spent sitting, and the fact that it does not capture all behaviours classified as sedentary, i.e. corresponding to an energy expenditure of $<1.5$ METs. There is thus a risk that the sitting time item from the IPAQ does not fully represent the actual sedentary time spent by the persons with RA included in the present study. In addition, studies comparing accelerometer and IPAQ data in persons with RA describe them to report less sedentary time and more highintensity physical activity than when objectively assessed [47, 48]. Nevertheless, IPAQ was originally designed for population surveillances that allow for comparisons with other populations, and the sitting time item has acceptable measurement properties [32]. A combination of selfreports and accelerometers is recommended to reach high specificity in measurement [47, 48], but is unfortunately not yet feasible in epidemiological studies. Another limitation to our study is the lack of disease activity data, which were only available from the SRQ at a matching point in time for less than $50 \%$ of the study sample. It can thus not be excluded that disease activity contributes in explaining the assessed health outcomes, although this has not been the case in all studies on RA using multivariate approaches $[49,50]$. The external validity of the study may be threatened by the small, but systematically skewed, data towards poorer health in the non-responders. Our results may, therefore, not be valid for an RA population with poorer health.

\section{Conclusions and implications for future studies}

The present study is, to the best of our knowledge, the first large-sample study reporting sedentary time in adults with RA as separated from time spent physically active. Daily sedentary time was low and did not contribute to explaining the variation in perceived health and activity limitation. Instead, these outcomes were primarily explained by self-reported pain, indicating an increased focus on pain in the management of RA. Future prospective studies should test the hypothesis based on the current study, i.e. that sedentary time does not explain variation in perceived health and activity limitation in people with RA. Such studies may benefit from measurement refinements, e.g. the use of accelerometers combined with information on context, type, and duration of sedentary behaviours per se.

Acknowledgements We would like to thank the rheumatology clinics at Danderyd Hospital, Stockholm, Karolinska University Hospital, Solna and Huddinge, Linköping University Hospital, Linköping and Norrköping, Mälarsjukhuset, Eskilstuna, Östersund Hospital, Sunderby Hospital, Luleå, and the SRQ registers for their generous provision of data for our study.

Funding This work was supported by the Swedish Research Council, Combine Sweden, the Swedish Rheumatism Association, the Forte foundation, the Strategic Research Area in Care Research, and the National Postgraduate School of Health Care Sciences at Karolinska Institutet, Stockholm, Sweden.

\section{Compliance with ethical standards}

Conflict of interest $\quad$ Author Demmelmaier, Åsenlöf, Bergman, Nordgren and Opava declare they have no conflict of interest.

Ethical approval All procedures performed in studies involving human participants were in accordance with the ethical standards of the institutional research committee (Stockholm Regional Review Board no 2010/1232-31/1) and with the 1964 Helsinki declaration and its later amendments or comparable ethical standards.

Informed consent Informed consent was obtained from all individual participants included in the study.

Open Access This article is distributed under the terms of the Creative Commons Attribution 4.0 International License (http:// creativecommons.org/licenses/by/4.0/), which permits unrestricted use, distribution, and reproduction in any medium, provided you give appropriate credit to the original author(s) and the source, provide a link to the Creative Commons license, and indicate if changes were made.

\section{References}

1. Owen N, Healy GN, Matthews CE, Dunstan DW (2010) Too much sitting: the population health science of sedentary behavior. Exerc Sport Sci Rev 38:105-113

2. Pate RR, O'Neill JR, Lobelo F (2008) The evolving definition of "Sedentary". Exerc Sport Sci Rev 36:173-178

3. Bankoski A, Harris TB, McClain JJ, Brychta RJ, Caserotti P, Chen KY et al (2011) Sedentary activity associated with metabolic syndrome independent of physical activity. Diabetes Care 34:497-503

4. Healy GN, Matthews CE, Dunstan DW, Winkler EAH, Owen N (2011) Sedentary time and cardio-metabolic biomarkers in US adults: NHANES 2003-06. Eur Heart J 32:590-597.

5. Koster A, Caserotti P, Patel KV, Matthews CE, Berrigan D, Van Domelen DR et al (2012) Association of sedentary time with mortality independent of moderate to vigorous physical activity. PLoS One 7:e37696 
6. Pedersen BK (2009) The diseasome of physical inactivityand the role of myokines in muscle-fat cross talk. J Physiol 587:5559-5568

7. Prioreschi A, Makda MA, Tikly M, McVeigh JA (2015) Habitual physical activity, sedentary behaviour and bone health in rheumatoid arthritis. Int J Sports Med 10:10

8. Gilbert AL, Lee J, Ma M, Semanik P, DiPietro L, Dunlop D et al (2015) Comparison of subjective and objective measures of sedentary behavior using the Yale Physical Activity Survey and accelerometry in patients with rheumatoid arthritis. J Phys Act Health. doi:10.1123/jpah.2015-0176

9. Khoja SS, Almeida GJ, Wasko MC, Terhorst L, Piva SR (2016) Light intensity physical activity is associated with lower cardiovascular risk factor burden in rheumatoid arthritis. Arthritis Care Res 68:424-431

10. Bauman A, Ainsworth BE, Sallis JF, Hagströmer M, Craig CL, Bull FC et al (2011) The descriptive epidemiology of sitting: A 20-country comparison using the International Physical Activity Questionnaire (IPAQ). Am J Prev Med 41:228-235

11. Matthews CE, Chen KY, Freedson PS, Buchowski MS, Beech BM, Pate RR et al (2008) Amount of time spent in sedentary behaviors in the United States, 2003-2004. Am J Epidemiol 167:875-881

12. Hagströmer M, Kwak L, Oja P, Sjöström M (2015) A 6 year longitudinal study of accelerometer-measured physical activity and sedentary time in Swedish adults. J Sci Med Sport 18:553-557

13. Dunstan DW, Howard B, Healy GN, Owen N (2012) Too much sitting — a health hazard. Diabetes Res Clin Pract 97:368-376

14. Rand D, Eng JJ, Tang PF, Jeng JS, Hung C (2009) How active are people with stroke? Use of accelerometers to assess physical activity. Stroke 40:163-168

15. Lee J, Chang RW, Ehrlich-Jones L, Kwoh CK, Nevitt M, Semanik PA et al (2014) Sedentary behavior and physical function: objective evidence from the osteoarthritis initiative. Arthritis Care Res 22:22432

16. Cieza A, Stucki G (2005) Understanding functioning, disability, and health in rheumatoid arthritis: the basis for rehabilitation care. Curr Opin Rheumatol 17:183-189

17. Cieza A, Ewert T, Ustun TB, et al (2004) Development of ICF Core Sets for patients with chronic conditions. J Rehabil Med Suppl 44:9-11

18. Pollard L, Choy EH, Scott DL (2005) The consequences of rheumatoid arthritis: quality of life measures in the individual patient. Clin Exp Rheumatol 23:S43-S52

19. Scott DL, Smith C, Kingsley G (2005) What are the consequences of early rheumatoid arthritis for the individual? Best Pract Res Clin Rheumatol 19:117-136

20. Metsios GS, Stavropoulos-Kalinoglou A, Kitas GD (2015) The role of exercise in the management of rheumatoid arthritis. Expert Rev Clin Immunol 15:1-10

21. Toussirot E (2010) Predictive factors for disability as evaluated by the health assessment questionnaire in rheumatoid arthritis: a literature review. Inflamm Allergy Drug Targets 9:51-59

22. Nordgren B, Fridén C, Demmelmaier I et al (2012) Long-term health-enhancing physical activity in rheumatoid arthritis. BMC Public Health 12:397

23. Demmelmaier I, Bergman P, Nordgren B, Jensen I, Opava C (2013) Current and maintained health-enhancing physical activity in rheumatoid arthritis-a cross-sectional study. Arthritis Care Res 65:1166-1176

24. Felson DT, Anderson JJ, Boers M, Bombardier C, Chernoff M, Fried B et al (1993) The American College of Rheumatology preliminary core set of disease activity measures for rheumatoid arthritis clinical trials. The Committee on Outcome Measures in Rheumatoid Arthritis Clinical Trials. Arthritis Rheum 36:729-740
25. Fries J, Spitz P, Kraines R, Holman H (1980) Measurement of patient outcome in arthritis. Arthritis Rheum 23:137-145

26. Ekdahl C, Eberhardt K, Andersson S, Svensson B (1988) Assessing disability in patients with rheumatoid arthritis. Use of a Swedish version of the Standford Health Assessment Questionnaire. Scand J Rheumatol 17:263-271

27. Buer N, Linton SJ (2002) Fear-avoidance beliefs and catastrophizing: occurence and risk factor in back pain and ADL in the general population. Pain 99:485-491

28. EuroQoL Group [2015-09-03]. http://www.euroqol.org

29. IPAQ Group. [2015-09-03]. http://www.ipaq.ki.se

30. Craig C, Marshall A, Sjöström M, Bauman A, Booth M, Ainsworth B (2003) International Physical Activity Questionnaire: 12-country reliability and validity. Med Sci Sports Exerc 35:1381-1395

31. Tierney M, Fraser A, Kennedy N (2015) Criterion validity of the International Physical Activity Questionnaire Short Form (IPAQ-SF) for use in patients with rheumatoid arthritis: comparison with the SenseWear armband. Physiotherapy 101:193-197

32. Rosenberg D, Bull F, Marshall A, Sallis J, Bauman A (2008) Assessment of sedentary behavior with the International Physical Activity Questionnaire. J Phys Act Health 5:S30-S44

33. Hubbard EA, Motl RW, Manns PJ (2015) The descriptive epidemiology of daily sitting time as a sedentary behavior in multiple sclerosis. Disabil Health J 8:594-601

34. Wullems JA, Verschueren SM, Degens H, Morse CI, Onambele GL (2016) A review of the assessment and prevalence of sedentarism in older adults, its physiology/health impact and non-exercise mobility counter-measures. Biogerontology. doi:10.1007/s10522-016-9640-1

35. Marshall SJ, Merchant G (2013) Advancing the science of sedentary behavior measurement. Am J Prev Med 44:190-191

36. Thomsen T, Beyer N, Aadahl M, Hetland ML, Løppenthin K, Midtgaard J et al (2015) Sedentary behaviour in patients with rheumatoid arthritis: a qualitative study. Int J Qual Stud Health Well-being. doi:10.3402/qhw.v10.28578

37. Bauer UE, Briss PA, Goodman RA, Bowman BA (2014) Prevention of chronic disease in the 21st century: elimination of the leading preventable causes of premature death and disability in the USA. Lancet 384:45-52

38. Kohl HW 3rd, Craig CL, Lambert EV, Inoue S, Alkandari JR, Leetongin $\mathrm{G}$ et al (2012) The pandemic of physical inactivity: global action for public health. Lancet 380:294-305

39. Semanik PA, Chang RW, Dunlop DD (2012) Aerobic activity in prevention and symptom control of osteoarthritis. PM\&R 4:S37-S44

40. Loppenthin K, Esbensen BA, Ostergaard M, Jennum P, Tolver A, Aadahl $M$ et al (2015) Physical activity and the association with fatigue and sleep in Danish patients with rheumatoid arthritis. Rheumatol Int 7:7

41. Rodríguez Huerta MD, Trujillo-Martín MM, Rúa-Figueroa Í, Cuellar-Pompa L, Quirós-López R, Serrano-Aguilar P (2016) Healthy lifestyle habits for patients with systemic lupus erythematosus: a systemic review. Semin Arthritis Rheum 45:463-470

42. Desveaux LPM, Beauchamp MMP, Goldstein RMDF, Brooks DPM (2014) Community-based exercise programs as a strategy to optimize function in chronic disease: a systematic review. Med Care 52:216-226

43. Cherubino P, Sarzi-Puttini P, Zuccaro SM, Labianca R (2013) The management of chronic pain in important patient subgroups. Clin Drug Investig 32:35-44

44. van Laar M, Pergolizzi JV, Mellinghoff H-U, Merchante IM, Nalamachu S, O'Brien J et al (2012) Pain Treatment in arthritisrelated pain: beyond NSAIDs. Open Rheumatol J 6:320-330 
45. Scascighini L, Toma V, Dober-Spielmann S, Sprott H (2008) Multidisciplinary treatment for chronic pain: a systematic review of interventions and outcomes. Rheumatology 47:670-678

46. Sarzi-Puttini P, Atzeni F, Clauw DJ, Perrot S (2015) The impact of pain on systemic rheumatic diseases. Best Pract Res Clin Rheumatol 29:1-5

47. Yu CA, Rouse PC, Veldhuijzen Van Zanten JJ, Ntoumanis N, Kitas GD, Duda JL et al (2015) Subjective and objective levels of physical activity and their association with cardiorespiratory fitness in rheumatoid arthritis patients. Arthritis Res Ther 17:59
48. Igelström H, Emtner M, Lindberg E, Åsenlöf P (2013) Level of agreement between methods for measuring moderate to vigorous physical activity and sedentary time in people with obstructive sleep apnea and obesity. Phys Ther 93:50-59

49. Gåfvels C, Hägerström M, Nordmark B, et al (2014) What predicts negative effects of rheumatoid arthritis? A follow-up two years after diagnosis. SpringerPlus 3:118

50. Nicassio PM, Kay MA, Custodio MK et al (2011) An evaluation of a biopsychosocial framework for health-related quality of life and disability in rheumatoid arthritis. J Psychosom Res 71:79-85 